\title{
Nuevas recomendaciones para el rastreo de cáncer colorrectal
}

\author{
New recommendations for colorrectal cancer screening
}

\section{Comentado de:}

Helsingen et al. BMJ 2019; 367:I5515. PMID: $31578196^{1}$

\section{Introducción}

Las actualizaciones recientes de los últimos 15 años de los ensayos clínicos sobre el rastreo de cáncer de colon con sigmoidoscopia proporcionan nuevas pruebas sobre la efectividad de la detección temprana del cáncer colorrectal. Basándose en esta nueva evidencia se ha formulado las preguntas: El rastreo del cáncer colorrectal ¿marca una diferencia importante en los resultados de salud en las personas que lo inician entre los $50 \mathrm{y}$ los 79 años? ¿Qué opción de detección es la mejor?

\section{Métodos}

Un panel de guía que incluyó pacientes, médicos, expertos de contenido y metodólogos produjo estas recomendaciones utilizando el enfoque GRADE y cumpliendo con los estándares para obtener pautas confiables. Se realizó una revisión sistemática de ensayos clínicos de detección de cáncer colorrectal y de modelos de microsimulación para informar al panel acerca de los beneficios y los daños del rastreo de esta enfermedad a 15 años. El panel también revisó los problemas prácticos y la carga de cada opción de detección. Basado en su propia experiencia, el panel estimó la magnitud del beneficio que los miembros típicos de la población valorarían para optar por realizar el rastreo, y utilizó estos umbrales de beneficios para informar sus recomendaciones. Esta guía analizó la evidencia e hizo recomendaciones sobre el rastreo del cáncer colorrectal con cuatro opciones: prueba inmunoquímica fecal (FIT, por sus iniciales en inglés) anual, FIT cada dos años, una sigmoidoscopia única o una colonoscopia única.

\begin{abstract}
Resultados
Evidencia

En general, hubo una incertidumbre sustancial (evidencia de baja certeza) con respecto a los beneficios a 15 años, las cargas y los daños del cribado. Las mejores estimaciones sugirieron que las cuatro opciones de detección dieron como resultado reducciones similares de mortalidad por cáncer colorrectal. La estrategia de rastreo con FIT cada dos años puede tener poco o ningún efecto sobre la incidencia de cáncer durante 15 años, mientras que la FIT anual, la sigmoidoscopia y la colonoscopia pueden reducir la incidencia de cáncer, aunque para la FIT la reducción de la incidencia es pequeña en comparación con las otras estrategias. Los eventos adversos cardiovasculares y gastrointestinales graves relacionados con estas prácticas son poco frecuentes. La magnitud de los beneficios depende del riesgo individual, mientras que los daños y las cargas están menos asociados con el riesgo de cáncer.
\end{abstract}

\section{Recomendaciones}

Las recomendaciones de esta guía se aplican a adultos de 50 a 79 años sin detección previa ni síntomas de cáncer colorrectal y una esperanza de vida de, al menos, 15 años.

Para las personas con un riesgo estimado de cáncer colorrectal de 15 años por debajo de $3 \%$, la guía sugiere que no se realicen pruebas de detección (recomendación débil). Para las personas con un riesgo estimado de 15 años superior a $3 \%$, sugiere la detección con una de las cuatro opciones: FIT anual, FIT cada dos años, una sola sigmoidoscopia o una sola colonoscopia (recomendación débil, ver Tabla 1). Para la estimación del riesgo individual de desarrollar cáncer colorrectal se sugiere utilizar la calculadora QCancer ${ }^{\circledR}$ (disponible en https://qcancer.org/15yr/col orectal $^{2}$ ).

Tabla 1. Recomendaciones principales de la guía. Nota: Métodos disponibles: prueba inmunoquímica fecal (FIT) anual, FIT cada dos años, una sigmoidoscopia única o una colonoscopia única.

\begin{tabular}{|l|l|}
\hline \multicolumn{1}{|c|}{ Escenario } & \multicolumn{1}{|c|}{ Recomendación } \\
\hline $\begin{array}{l}\text { Personas con un riesgo estimado a } 15 \text { años de cáncer colorrectal de } \\
\mathbf{3} \% \text { o superior }\end{array}$ & $\begin{array}{l}\text { Realizar rastreo con alguno de los cuatro métodos disponibles } \\
\text { (recomendación débil) }\end{array}$ \\
$\begin{array}{l}\text { Personas con un riesgo estimado a } 15 \text { años de cáncer colorrectal } \\
\text { menor a } 3 \%\end{array}$ & $\begin{array}{l}\text { No realizar rastreo } \\
\text { (recomendación débil) }\end{array}$ \\
\hline
\end{tabular}

\section{Recomendación final}

Con base en los beneficios, daños y cargas de la detección, el panel infirió que las personas más informadas con un riesgo de cáncer colorrectal de 15 años de $3 \%$ o más tienen mayor probabilidad de elegir el rastreo, mientras que la mayoría de las personas con un riesgo de menos de $3 \%$ es más probable que rechacen realizar esta práctica preventiva. Dados los diferentes valores y preferencias de las personas, la atención óptima requerirá una toma de decisiones compartida con los pacientes.

Fuente de financiamiento/Conflictos de interés:: Esta guía no tuvo financiamiento. Los autores declaran no tener conflictos de interés. 


\section{Comentario}

La guía de práctica clínica resumida ${ }^{1}$ cumple criterios de calidad según la herramienta AGREE I $^{3}$ : determina objetivos y alcances de las recomendaciones, realiza una amplia convocatoria de diversos miembros en su panel y utiliza criterios rigurosos para la síntesis de evidencia y formulación de recomendaciones, incluyendo el enfoque GRADE ${ }^{4}$ con una adecuada independencia editorial y libre de conflictos de intereses. Sin embargo, los autores aclaran que esta guía no toma una perspectiva de salud pública, incluyendo aspectos relacionados con el uso de recursos, equidad o factores relacionados con la implementación local (dominio 5 de la herramienta AGREE II), por lo cual cada sistema de salud debería realizar sus consideraciones a la hora de implementar sus recomendaciones.

Lo más innovador de esta guía es la incorporación explícita de los valores y preferencias de los pacientes, valorando la importancia relativa de los beneficios y los daños de cada método diagnóstico. Este aspecto suele estar pobremente desarrollado en las guías de práctica clínica, y existen distintas metodologías para su abordaje ${ }^{5}$. A diferencia de las recomendaciones estándar de la Fuerza de Tareas Preventivas de los EE.UU. (USPSTF por sus iniciales en inglés) y del Ministerio de Salud de la Argentina ${ }^{6,7}$, que recomiendan el rastreo a partir de los 50 años de edad, en esta nueva guía se recomienda incorporar el riesgo individual de desarrollar cáncer en la toma de decisiones con los pacientes, junto a los beneficios y los daños de esta práctica preventiva. Considerando que el riesgo de $3 \%$ a 15 años en población general se lograría aproximadamente a los 60 años $^{2}$, esta nueva guía desaconsejaría el rastreo en menores de esa edad. Aunque el método que utilizaron para evaluar los valores y las preferencias tiene limitaciones (fue una valoración cuantitativa delimitada al panel), esta innovadora guía plantea la necesidad de explorar las preferencias individuales y poblacionales en relación al rastreo de cáncer colorrectal.

\section{Conclusiones del comentador}

Las nuevas recomendaciones para el rastreo de cáncer colorrectal indican que la decisión de inicio de rastreo sería sensible a los valores y las preferencias de las personas, por lo cual es esencial que se planteen las opciones de tamizaje (incluyendo la opción de no realizar esta práctica) con un abordaje de toma de decisiones compartida.

Juan Victor Ariel Franco [ Servicio de Medicina Familiar y Comunitaria, Hospital Italiano de Buenos Aires, Departamento de Investigación, Instituto Universitario Hospital Italiano juan.franco@hospitalitaliano.org.ar ]

Franco JVA Nuevas recomendaciones para el rastreo de cáncer colorrectal. Evid Actual Pract Ambul. 2019;22(4):e002024. Comentado de: Helsingen LM, et al. Colorectal cancer screening with faecal immunochemical testing, sigmoidoscopy or colonoscopy: a clinical practice guideline. BMJ $2019 ; 367$ :I5515. PMID: 31578196

\section{Referencias}

1. Helsingen LM, Vandvik PO, Jodal HC, Agoritsas T, Lytvyn L, Anderson JC, et al. Colorectal cancer screening with faecal immunochemical testing, sigmoidoscopy or colonoscopy: a clinical practice guideline. Bmj. 2019;p. I5515. Available from: 10.1136/bmj.I5515.

2. QCancer ${ }^{\circledR}$ (15yr, colorectal) risk calculator; 2019. Available from: https://www.qcancer.org/15yr/colorectal/ [Last access: 2019-12-15].

3. Brouwers MC, Kho ME, Browman GP, Burgers JS, Cluzeau F, Feder G, et al. AGREE II: Advancing guideline development, reporting and evaluation in health care. Journal of Clinical Epidemiology. 2010;63(12):1308-1311. Available from: 10.1016/j.jclinepi.2010.07.001.

4. Alonso-Coello P, Schünemann HJ, Moberg J, Brignardello-Petersen R, Akl EA, Davoli M, et al. GRADE Evidence to Decision (EtD) frameworks: a systematic and transparent approach to making well informed healthcare choices. 1: Introduction. BMJ (Clinical Research Ed). 2016;353:i2016i2016.

5. Zhang Y, Coello PA, zek JB, Wiercioch W, Etxeandia-lkobaltzeta I, Akl EA, et al. Using patient values and preferences to inform the importance of health outcomes in practice guideline development following the GRADE approach. Health and Quality of Life Outcomes. 2017;15(1):52. Available from: 10.1186/s12955-017-0621-0.

6. USPSTF. Screening for Colorectal Cancer: US Preventive Services Task Force Recommendation StatementUSPSTF Recommendation Statement: Screening for Colorectal CancerUSPSTF Recommendation Statement: Screening for Colorectal Cancer. Jama. 2016;315(23):2564-2575. Available from: 10.1001/jama.2016.5989.

7. Gualdrini UA, lummato LE, Bidart ML, Instituto Nacional del Cáncer. Programa Nacional De Prevención Y Detección Temprana Del Cáncer Colorrectal Guía Para Equipos De Atención Primaria De La Salud; 2015. p. 15. 